Trinidad Bergero Miguel, Susana Asiain Vierge, Isolde Gorneman Schaffer, Francisco Giraldo Ansio, José Lara Montenegro, Isabel Esteva de Antonio y Marina Gómez Banovio

\title{
Una reflexión sobre el concepto de género alrededor de la transexualidad
}

RESUMEN: Transexualismo tratado desde un abordaje multidisciplinar en el que se ofrece un planteamiento desde distintas perspectivas.

PALABRAS CLAVE: Transexualismo, género, salud/enfermedad.
SUMMARY: Transsexualism needs a multidisciplinary approach. The aim of this article is to offer an exposition from different perspectives. KEY WORDS: Transsexualism, gender, health/ disease.

\section{Transexualidad. Definición. La concepción de la enfermedad. Modelo biomédico}

Sabemos que la transexualidad ha estado presente en todas las culturas y tiempos históricos de la Humanidad (1-4). Sus distintas formas de manifestación han estado moduladas por la respuesta social, donde destacan tanto los temas de aceptación e integración, como la posibilidad de recibir tratamiento.

La transexualidad es poco frecuente y es la forma más extrema de la disforia de género (5). Parece probable que las diferencias culturales puedan influir en las expresiones conductuales de estas personas.

Desde el enfoque sanitario de la clasificación de las enfermedades, la transexualidad es considerada un trastorno mental en la medida en que produce un sufrimiento significativo y una desventaja adaptativa importante. El umbral clínico se traspasa cuando la persona experimenta preocupación, incertidumbre por su identidad de género, llegando en ocasiones a ser esta experiencia tan intensa que se convierte en el aspecto central de la vida, lo cual obstaculiza el logro de una identidad de género libre de conflictos y en algunos casos, impide la normalización de la vida misma (5). Los criterios diagnósticos que se usan provienen de las clasificaciones internacionales de los trastornos mentales, establecidas por la Asociación de Psiquiatras Americanos y la Organización Mundial de la Salud. Estas clasificaciones siguen el modelo médico orientado a la clasificación, diagnóstico y teorización a partir de la recolección de datos biográficos y psicológicos $(6 ; 7)$. La calificación de patología mental ha sido cuestionada desde distintos sectores, y las asociaciones han advertidos que esta nomenclatura no debe ser usada con fines de estigmatización $(8 ; 9)$. Una de las dificultades más claras para establecer el diagnóstico, es la gran diversidad de formas, situaciones y vivencias con que se presenta y que no se ajustan a las concepciones binarias que manejamos respecto a lo que es ser un hombre o una mujer en nuestra sociedad. Tanto la CIE-10 como la 
DSM-IV en sus descripciones de la transexualidad, presentan importantes carencias y en muchos casos, resultan claramente insuficientes (5).

Algunos autores piensan que el pensamiento dualista en forma de oposiciones binarias (hombre/mujer) es una de las mayores trabas no sólo para la construcción de identidades y roles de género alternativos, sino también para cumplir el delicado cometido del proceso diagnóstico especificado como una de las tareas más importantes del profesional de salud mental en los protocolos internacionales (8; 9). DSM IV (6) lo designa como Trastorno de la Identidad Sexual (F64.x) y exige al menos dos componentes de los siguientes que deben estar presentes a la hora de efectuar el diagnóstico:

Criterio A: El individuo se identifica de un modo intenso y persistente con el otro sexo, lo cual constituye el deseo de ser, o la insistencia en que uno es, del otro sexo.

Criterio B: Esta identificación con el otro sexo no es únicamente el deseo de obtener las supuestas ventajas relacionadas con las costumbres culturales. Deben existir también pruebas de malestar persistente por el sexo asignado o un sentido de inadecuación en el papel de su sexo.

Criterio C: El diagnóstico no debe establecerse si el individuo padece una enfermedad física intersexual.

Criterio D: Deben existir pruebas de malestar clínicamente significativo o deterioro social, laboral o de otras áreas importantes de la actividad del individuo. También se especifica la orientación sexual: hacia varones, mujeres, ambos sexos o ninguna atracción sexual. La Organización Mundial de la Salud, en su décima revisión (CIE-10) CIE-10 (7) lo denomina Transexualismo (F64) y lo define como «el deseo de vivir y ser aceptado como un miembro del género opuesto, por lo general acompañado por el deseo de modificar mediante métodos hormonales o quirúrgicos el propio cuerpo para hacerlo lo más congruente posible con el género preferido». Se requiere la presencia de identidad transexual persistentemente durante al menos dos años. No se trata de un síntoma de otro trastorno mental, tal como Esquizofrenia, ni secundario a una anomalía cromosómica. En ambas definiciones, se establece la necesidad de objetivar una divergencia entre el desarrollo de un sexo morfoanatómico y fisiológico perteneciente a un género, y la construcción de una identidad sexual de signo contrario. En el caso de la CIE-10, se alude específicamente a que por lo general, se presenta acompañado por el deseo de someterse a tratamiento quirúrgico u hormonal para lograr que el cuerpo concuerde lo más posible con el género preferido (7). Describiéndolo de esta manera, la CIE-10 sigue la línea de autores clásicos sobre el tema, como Person y Ovesey que en 1974 definían el transexualismo como la resolución de una reasignación del sexo por medio de las hormonas y de la cirugía en personas biológicamente normales (10). 
Stoller, otro autor clásico, había introducido con anterioridad, una matización respecto a este punto definiendo el Transexualismo como «la convicción de un sujeto, biológicamente normal, de pertenecer al otro sexo. En el adulto, a esta creencia le acompaña en nuestros días la demanda de intervención quirúrgica y endocrinológica para modificar la apariencia anatómica en el sentido del otro sexo» (11).

Hay una diferencia entre las definiciones de Person y Ovesey y Stoller. Este último es más contextual, y tiene en cuenta los aspectos del momento histórico en los que describe las características de la Transexualidad, considerando que la necesidad de las intervenciones sanitarias no son inherentes a la definición de la Transexualidad, sino producto del momento actual, en el que el progreso de los conocimientos y de las técnicas permiten la utilización de terapias hormonales y quirúrgicas para lograr un cambio morfoanatómico y hormonal impensable en otras etapas de la humanidad (5).

Al hilo de esta cuestión, cabe preguntarse: ¿Qué ocurría cuando no existía la posibilidad de hacer una intervención de esta magnitud sobre el cuerpo?

Harry Benjamin en su obra El fenómeno transexual dijo: «Dado que es evidente, pues, que la mente del transexual no puede ser cambiada en su falsa orientación de género, es lógico y justificable intentar lo opuesto, ajustar el cuerpo a la mente» (12).

Esta frase refleja la ideología biomédica respecto al sexo y a la identidad de género. Por una parte se descubre que en el tratamiento del transexual subyace un marcado dualismo entre el cuerpo y la mente, lo que posibilita ajustar el sexo, que en este caso se iguala a la realidad corporal, a las definiciones normativas de género. Es decir, la identidad de género se entiende como inmutable y constante a lo largo del tiempo mientras que el sexo se convierte en maleable ayudado por la tecnología médica mediante métodos hormonales, quirúrgicos, transformando así la realidad corporal en artefacto para alcanzar así una identidad integrada.

Con estos ejemplos de definiciones de la Transexualidad, tomamos conciencia de la afirmación de algunos autores que sostienen que en el Transexualismo «la marca en el cuerpo se juzga indispensable, y que en la petición de reasignación de sexo que hacen estas personas, se establece una primacía de lo biológico sobre la palabra, sobre lo simbólico» (13). Y esto es así porque en nuestra cultura hay una fuerte insistencia en la corporeización de los estereotipos de género, ese conjunto de ideas simples pero fuertemente arraigadas en la conciencia, que escapan al control de la razón y que definen lo que es masculino o femenino en un contexto social determinado $(14,15)$. La fuerza de estos estereotipos de género no es solamente psicológica, sino que están dotados de una realidad material que contribuye a consolidar las condiciones sociales y económicas dentro de las cuales se generan (16).

Las descripciones de la Transexualidad que manejamos, provienen de un modelo de salud y enfermedad biomédico muy interesante, de gran utilidad, siem- 
pre que no perdamos de vista que este modelo no es universal ni ahistórico. Las formulaciones de la salud y la enfermedad de la Medicina están también determinadas culturalmente (17). Muchas veces no tenemos en cuenta los aspectos ideológicos o históricos que intervienen en los mecanismos por los que se determina en un momento dado, qué es patológico o qué no lo es. Sin más recordemos el ejemplo de la homosexualidad considerada como trastorno hasta hace poco (18).

El modelo actual, la biomedicina, es un sistema basado en el método científico, pero al mismo tiempo, una institución social y un aparato ideológico-cultural y organizativo históricamente determinado (19).

La Medicina continúa su búsqueda en relación con la etiología de la transexualidad que actualmente va encaminada a la existencia de un trastorno en la diferenciación sexual del cerebro. Esta nueva línea de investigación ha sido acogida con entusiasmo por la comunidad médica, ya que significaría que la transexualidad pasaría a considerarse un trastorno biológico. Gooren, afirma: «Los transexuales podrían ser «rehabilitados», pasando de perturbados mentales a víctimas de un trastorno en la diferenciación sexual y los seguros médicos podrían ser (legalmente) obligados a cubrir los costos de la reasignación de sexo, como en otros casos de intersexualidad» (20).

\section{2. ¿Por qué produce tanto sufrimiento?}

Por muchas razones. La sociedad determina los límites simbólicos que indican quién está dentro y quién queda fuera del orden social. Sentirse incluido en uno de los polos de la dicotomía hombre-mujer, ofrece seguridad. La identidad es un proceso que permite a las personas ubicarse en el mundo. La identidad se piensa de forma ontológica. La configuración esencialista del pensamiento occidental tiende a naturalizar la identidad y a definirla como estructura sólida, nada cambiante. Decir: «soy mujer», sentirse un miembro de un grupo predeterminado socialmente, tranquiliza, ayuda a vivir sin dudas ni ansiedades.

Desde esta perspectiva se pueden entender los altos niveles de angustia que produce situarse fuera del modelo dicotómico que utilizamos cuando desde el punto de vista genético, hormonal y morfoanatómico se pertenece a un sexo y desde el punto de vista psicológico y social a otro (21). Nuestro modelo occidental no contempla un lugar para aquellas mujeres sociales que tienen genitales masculinos. La cirugía transexual subraya el modelo occidental de género, encarnando la polarización de género con base en los genitales y el cuerpo $(4 ; 22)$.

Otro motivo de sufrimiento radica en que por ahora, la solicitud de reasignación de sexo, sigue siendo una demanda individual, sin estatus social de acogida, de aquí la importante angustia que exponen las personas transexuales, la crisis de 
DEBATES E INFORMES

identidad sin precedentes de las que hablan algunos autores (13). Chiland afirma que las personas transexuales viven en un momento histórico en el que los conocimientos médicos y quirúrgicos «hacen posible cierto cambio de la apariencia, pero no la realización completa de su sueño: ser completamente el otro» (13). Continuando con los motivos de sufrimiento, al trabajar en este tema, se toma conciencia de que una de las dificultades más importantes es el aislamiento social que sufren muchas de estas personas (6) que repercute de una manera notable en el nivel de autoestima, ya que tienen que enfrentar consecuencias adversas por poseer un autoconcepto poco reforzado socialmente (22) además de contribuir a crear dificultades en la adaptación social produciendo interrupciones tempranas de la escolarización y dificultades en el ámbito laboral. Las dificultades de socialización, del desarrollo escolar y laboral llevan, en ocasiones, a la práctica de trabajos marginales. Asimismo, la preocupación por su aspecto físico y los cambios que quieren conseguir, para adaptarse al otro sexo y disminuir el malestar que les produce su sexo biológico, se convierte en una actividad absorbente que ocupa gran parte de su tiempo y que entorpece aun más la adaptación al medio (6). Estas dificultades a lo largo del ciclo vital, dan lugar a la necesidad de exploración de la comorbilidad psíquica, como parte indispensable del proceso diagnóstico.

\section{El punto de vista de la Antropología Social}

La Medicina se ha ocupado del estudio de la transexualidad, y también la Psicología, la Sociología y la Antropología han investigado acerca de la misma pero desde perspectivas diferentes.

La Antropología Social ha aportado una visión amplia del concepto de sexualidad. Ha tratado de determinar los criterios básicos de diferenciación entre los sexos, las especificidades de las representaciones que orientan los comportamientos sexuales y cómo esas representaciones son vividas por los actores sociales en situaciones y contextos socioculturales siempre concretos (4).

Tratando de dar respuestas a estas complejas preguntas, la Antropología Social ha encontrado en el camino a personas transexuales, travestistas, transgéneros, etc. Es interesante la perspectiva antropológica, que utiliza el concepto de transgénero: individuos, comportamientos y grupos que presentan divergencias con los roles de género duales más tradicionales, ya que traspasan las fronteras de identidad genérica comúnmente asignadas» (15). Este concepto incluye a un variado grupo de personas andróginas o transexuales, teniendo en cuenta que no es determinante el deseo de reasignación quirúrgica de genitales ya que éstos no tienen por qué considerarse la esencia de la construcción de la identidad de género (15). Para la Antropología Social, la transexualidad se concibe no como una enfer- 
medad individual, sino como un producto resultante de aplicar un modelo determinado de construcción sociocultural a la identidad sexual y de género, en un contexto concreto. En el modelo que manejamos, ser hombre es no ser mujer. Se basa en la dicotomía. Se trata de un modelo que elimina la graduación y el matiz en un tema de gran complejidad y diversidad.

Desde la Antropología, la transexualidad se ha incluido dentro de la comunidad transgenérica, formada a su vez por otras personas que presentan identidades que o bien no se ajustan al binarismo sexual y de género imperante en la sociedad, o que transitan continuamente entre un polo y su opuesto. Transgenerismo sería el término que mejor expresaría la continuidad y pluralidad en las variaciones de género identitarias.

La Antropología Social, además tiene en cuenta el protagonismo individual y la capacidad de decisión de cada sujeto a la hora de aceptar o rechazar los constreñimientos socioculturales del momento histórico en que vive, así como de desarrollar diferentes estrategias respecto a ellos. Esta interacción entre individuo y sociedad/cultura nos lleva al concepto de intersubjetividad.

Según A. Martín Casares, la tendencia europea al binarismo, dividiendo a los seres humanos en masculino y femenino, y excluyendo otras posibilidades de identidad de género, condiciona en gran medida nuestra percepción de en qué consiste la esencia humana (15). Otra aportación importante de la Antropología Social es la revisión de nuestro modelo que da por supuestas la inmutabilidad y la estabilidad de la identidad de género, así como su esencialidad, ideas asociadas al modelo que propone la existencia diferenciada de los géneros masculino y femenino (21). Lo que una persona «es» se refiere no sólo a lo biológico, sino que también «es» el resultado de los sucesivos roles que desempeña en la vida cotidiana y que irán cambiando a lo largo de su trayectoria vital. Desde esta perspectiva la identidad es un proceso y no una estructura y sólo puede entenderse en términos históricos (21). Básicamente y de modo breve, podemos decir que los paradigmas más importantes desarrollados por la Antropología Social, de interpretación de la transexualidad englobada dentro de la comunidad «transgénero», son tres (4): a) Como expresión de un tercer género. b) Como reforzamiento de las identidades genéricas. c) Como género preformativo.

\subsection{Como expresión de un tercer género. Géneros múltiples}

Desde esta perspectiva, el transgénero es considerado una tercera posibilidad en la organización y representación de género. Esta perspectiva se encuentra sobre todo en las etnografías referidas a culturas no occidentales, como por ejemplo en el estudio de los berdache por Kroeber (23) y últimamente en estudios antropológicos en la sociedad occidental. 
DEBATES E INFORMES

Este tercer género, surge de una reinterpretación del sexo y del género que impugna el dimorfismo sexual. Según este paradigma, las categorías varón o mujer no son universalmente válidas. Las personas «transgéneros» desbordan el binarismo aplicado al sexo y al género y no pueden ser interpretadas bajo esos términos, por tanto quedan relegados a «lo otro», a lo que no puede ser interpretado a partir del dimorfismo sexual (15).

En esta línea Roscoe propone un paradigma de géneros múltiples donde el género sea potencialmente autónomo del sexo ya que no en todas las culturas los marcadores del sexo son vistos como naturales y contrapuestos a un dominio distinto de lo cultural» (4).

Este modelo surge a partir de la reinterpretación de los conceptos de género y sexo basados en el paradigma del dimorfismo sexual. Las categorías hombre o mujer basadas en criterios anatómicos, en primer lugar no son universales y en segundo lugar, no son conceptos válidos para un sistema de clasificación de género (4). En determinados lugares y momentos históricos de la humanidad, las sociedades y personas han ido construyendo situaciones que transcienden y desbordan las rígidas coordenadas que delimitan las categorías hombre/mujer y masculino/femenino (4).

Desde este punto de vista se pueden englobar los hijras de la India y Pakistán (24) o los eunucos de los harenes orientales (25) o los inuitas o los berdaches (4) por poner algunos ejemplos. Se trata de categorías o estatus reconocidos culturalmente y que no pueden ser explicados por un modelo dualista. Las categorías de género que manejamos se basan a menudo en percepciones de las diferencias anatómicas y fisiológicas entre los cuerpos, pero no hay que olvidar que estas percepciones están siempre mediatizadas por categorías y significaciones culturales (23).

Estudios de distintas culturas, revelan una gran variabilidad en los rasgos socioculturales de los roles sexuales y en las creencias concernientes al cuerpo y a lo que constituye el sexo. No todas las culturas reconocen los mismos marcadores anatómicos y no todas perciben las marcas anatómicas como naturales y contrapuestas a un dominio distinto de lo cultural (4). Las personas transgéneros cuestionan el sistema de género que utilizamos, los límites de la polaridad de nuestro modelo. Sugieren un continuum de masculinidad y feminidad, una renuncia al género alineado con los genitales, el cuerpo, etc. (4). Es a finales del siglo XX cuando el modelo de los géneros múltiples anteriormente reservado para el estudio de las culturas no occidentales, va ganando presencia en las sociedades occidentales como marco para los debates sobre sexualidad e identidad de género. La existencia de individuos y grupos en nuestras sociedades que comparten y combinan determinadas propiedades y características que los excluyen de las categorías varón o mujer, es el motivo central de dicho paradigma. La propuesta es de géneros supernumerarios o géneros múltiples. «El tercer género aparece como el lugar para la construcción de múltiples identidades que recomponen dimensiones cuya 
vinculación se desnaturaliza y que por lo mismo, pueden escapar a las normas socialmente impuestas» (4).

\subsection{Como reforzamiento de las identidades genéricas}

Desde esta perspectiva, se cuestiona la categoría tercer género como un tercer estatus sexual y como otra posibilidad en la organización de las representaciones de género. La pregunta que se formula desde este paradigma es si es posible pensar en un género con total independencia de la diferencia sexual.

Desde esta posición, las personas transexuales ven al género como algo rígidamente demarcado y excluyente: masculinidad y feminidad, reflejando en sus cambios y en su solicitud de reasignación, los roles de géneros tradicionales, autoexcluyentes entre sí. La identidad transexual solicitando ser exactamente una réplica del otro sexo, sostiene el paradigma occidental basado en la existencia de dos sexos opuestos y de conductas, temperamentos, emociones y orientaciones sexuales constituidas sobre la base de una polaridad biológica. Esta oposición está representada por los genitales, símbolos de las diferencias reproductivas y base para la asignación del sexo biológico (4).

En el caso de las transexuales hombre-a-mujer, observamos que algunas de estas personas reproducen una estereotipada visión de la mujer que habitualmente ha sido usada para oprimirla (4).

\subsection{Como género performativo}

La tercera hipótesis tiene una perspectiva deconstructivista. Coincide con el paradigma múltiple de género en que el transgénero desafía la concepción binaria, la cual se considera reduccionista y pone en cuestionamiento las categorías de masculino y femenino, pero lejos de buscar géneros múltiples, busca la deconstrucción de la categoría misma de género. Esto se explica porque al haberse entendido el género como una construcción cultural tan ligada al sexo, ambos resultan tener una relación mimética en la que la diferencia entre ambos carece de sentido.

Butler propone el redespliegue de las perfomances de género -aquellas conductas y actividades que producen el género en la vida diaria y construyen como varones y mujeres a las personas implicadas en ellas- a través de repeticiones paródicas que pongan en evidencia el carácter performativo (como opuesto a expresivo) del género. Según esta autora, esas repeticiones desestabilizarían las nociones recibidas sobre la naturalidad del género como el núcleo de la identidad, iluminando al mismo tiempo, la relación artificial del género con los cuerpos y las sexualidades (26). 
DEBATES E INFORMES

Las personas transvestistas parecen integrar ese colectivo de personas con categorías desordenadas, formando parte del conjunto de espectros prohibidos por nuestra perspectiva que produce géneros inteligibles y perfectamente demarcados. Para Butler, los travestis son ejemplos de la repetición paródica del género en orden a subvertir sus significados en nuestra cultura. Parecen denunciar a través de sus autorepresentaciones, el hecho de que en realidad, el género no es otra cosa que la construcción mimética del sexo (26). Pero hay que entender bien a esta autora que advierte que performatividad de género no es teatralidad. A diferencia del rol teatral, el género no es elegido (26).

\section{4. ¿Qué mecanismos intervienen en la construcción de la identidad?}

A estas alturas estamos lejos de pensar que la identidad se funda solamente en la biología. El tema es complejo y hay que evitar posiciones reduccionistas que no tengan en cuenta otros aspectos como los psicológicos, sociales, contextuales. El entorno social, los valores con relación a lo masculino y femenino en una sociedad determinada, condicionan en gran medida nuestra conducta y nuestras actitudes, indican un modelo determinado a seguir, pautando entre otras características, los ideales de estatura y peso, los mecanismos de conservación, el tipo de alimentación, los cuidados estéticos, vestimentas, las características de las manifestaciones emocionales propios de uno u otro sexo. ¿Quiénes determinan el modelo imperante? Hasta la Revolución Francesa, el poder para definir la realidad social estaba en manos de la Iglesia. La Iglesia primero, la ciencia después y el mercado ahora son centrales en el proceso de construcción de las identidades sociosexuales. Olga Viñuales dice que los y las protagonistas de las disidencias sexuales, pecaron primero, enfermaron después y son hoy en día, consumidores de diferencia (27). Es difícil sustraerse del todo al sentido común de una época y a ciertas imágenes demasiado pregnantes que imperan en un momento histórico de su desarrollo.

El sufrimiento de estas personas, está determinado por la dificultad o imposibilidad de cumplir el rol de género esperado de acuerdo al sexo biológicamente determinado. Como consecuencia puede producirse una búsqueda de cumplir estereotipos, cambio de sexo y feminidad extrema. La inculcación de la feminidad no se produce intersubjetivamente, sino que su búsqueda se dirige hacia la feminidad en abstracto, es decir hacia modelos ajenos a la experiencia cercana, convirtiéndose así en una búsqueda de estereotipos. En las personas transexuales se dificulta la aceptación social, se interrumpe y altera el proceso de construcción de la identidad, lo que favorece en ocasiones, el desarrollo de identidades estereotipadas. Se trataría de demostrar la identidad, hombre o mujer prototípico. La conformación de la identidad de las personas como hombres o mujeres forman parte de 
un proceso cotidiano de reafirmación, negación o discusión de una identidad en la que el cuerpo constituye un aspecto fundamental y en el que influyen de gran manera los modelos presentados como ideales en un momento histórico determinado a través de la publicidad, los medios de comunicación, el mundo del deporte, de la cultura, del arte, de la música, etc. (28).

La imagen corporal y el cuerpo individual y social son fundamentales en la construcción de la propia identidad y en la determinación de la pertenencia a los diferentes grupos. El cuerpo es modelado y construido conforme a las exigencias y normativas de la sociedad en la que vivimos (28).

Por eso es tan importante recordar que para entender esta compleja realidad, utilizamos modelos, metáforas que intentan capturarla, pero que no se reducen a ellas. Ni trabajando en el tema de la Transexualidad ni en ningún otro, debemos olvidar que los modelos que utilizamos para analizar cualquier situación, son construcciones de redes ideológicas que cazan, ordenan, organizan y otorgan significado a lo que ocurre «ahí fuera» y «aquí dentro» (29) y conceptos como sexo y género son construcciones, que no son universales ni ahistóricas. No hay que confundir el modelo con la realidad.

El modelo que usamos constituye una manera de simplificar la diversidad y hacerla más manejable. Es más fácil ejercer el control social a través de la uniformidad que de la diversidad. La diversidad implica conflicto, negociación, desorden. Así se determina la existencia de personas transexuales con un denominador común que simplifica una realidad muy heterogénea y variada. Aunque históricamente la idea del feminismo de la diferencia que considera que la diferencia sexual es el elemento fundamental en la constitución social y psicológica de las personas, ha sido muy interesante para resaltar las desigualdades y también resaltar el empoderamiento, creemos con Mari Luz Esteban (28) que no se debe tomar esta diferencia sexual como un principio, sino que en todo caso, debe ser tomada como una hipótesis de trabajo en la coyuntura social que vivimos. Las aportaciones que enfocan la cuestión desde la diferencia son indudablemente necesarias para entender el tema del género en general y para trabajar en la transexualidad en particular.

\section{Importancia del cuerpo}

Trabajando con personas transexuales, se toma conciencia en toda su dimensión la importancia del cuerpo como campo de la cultura. Al decir esto no sólo estamos afirmando que lo social se inscribe en el cuerpo, sino que el cuerpo es un «proceso material de interacción social» (30). El cuerpo tiene una dimensión biológica, pero también un lugar de intersección tanto del orden individual y psicológico, como social. 
Pero el aprendizaje del uso del cuerpo y de la imagen personal están determinados también por otros factores como son el sexo, el género, la edad, la clase social, la cultura, las creencias religiosas, la actividad laboral, etc. y es un mecanismo de retroalimentación y cambio porque las prácticas individuales y grupales en relación al cuerpo, influyen a su vez en definiciones sociales generales (28). La relación con personas transexuales nos ha hecho reflexionar acerca de las ambiguas y dinámicas fronteras que existen entre lo masculino y lo femenino, de la transgresión y desaparición de esas fronteras en relación a ciertas actividades físicas y sociales (28).

El trabajo con el cuerpo que llevan a cabo con la ayuda de los profesionales sanitarios que les asisten, les permiten una apropiación de sí mismas, permitiéndoles articular íntimamente trabajo corporal y proyecto de vida. El cuerpo es el elemento principal de este proceso, producto y agente, objeto y sujeto al mismo tiempo. El cuerpo es activo y autotransformante. Debemos tomar en cuenta la carnalidad de la experiencia humana (28). Desde este punto de vista, estas personas demuestran que lo corporal no es natural, sino que «siempre es construido social y políticamente». «El cuerpo es un lugar de discriminación, pero también de resistencia y contestación» (28). Las nuevas tecnologías, la utilización de distintos materiales y prótesis para modelar cuerpos e imitar imágenes corporales idealizadas, han introducido en nuestra sociedad occidental, la posibilidad de importantes cambios en él. Lo orgánico se combina con lo artificial. Naturaleza y sexualidad adquieren nuevos significados y conducen a formas distintas de percibirnos como seres humanos. Esta posibilidad recuerda el esfuerzo postestructuralista de D. Haraway cuando habla de los cuerpos reconstruidos y fabricados, cyborgs (31).

Las personas transexuales hablan de la intervención quirúrgica (mamoplastia, genitoplastia) como de un nuevo nacimiento, colocando expectativas a veces poco realistas y negando muchas veces la importancia de la vida anterior y de los vínculos y afectos establecidos, como así también negando una historia previa de ambigüedad y sufrimiento, confusión, dudas, períodos de transvestismo, transvestismo fetichista, etc. (21). Algunas de estas personas llegan pensando que deben posponer todos sus proyectos y acciones para después de la intervención quirúrgica, actuando con conductas de evitación ante situaciones para los demás, cotidianas y normalizadas, con lo cual se colocan en situación de desventaja con respecto a sus pares desde el punto de vista afectivo, laboral, educacional, etc. $(21 ; 32)$.

En nuestra experiencia hemos observado la presión que sufren algunos de nuestros pacientes para pasar necesariamente por la cirugía de genitales. La presión puede provenir tanto del medio familiar y social en el que se mueve (presión homogenizadora) como de otras personas transexuales que pueden mirar con desconfianza a los que no solicitan esta intervención. Se les explica que la genitoplastia no es imprescindible. Pero es aconsejable en aquellas personas en que existe una importante angustia asociada a la discordancia entre el género que sienten 
como suyo y su anatomía genital (21). Algunas de estas personas se muestran más eficaces a la hora de hablar del horror que les produce su cuerpo, mamas o genitales, que para hablar de lo que desean ser (32). Al mostrarse en el Test de la vida real como hombres o mujeres claramente identificables, logrando una buena aceptación del entorno, disminuyen los elevados niveles de ansiedad que presentan y de esta manera logran un mayor refuerzo social y les proporciona una mayor seguridad, mejorando el autoconcepto. La dimensión cognitiva, sensitiva, emocional, performativa y de acción social, se va entretejiendo y retroalimentando. La identidad siempre se produce en la interacción con los otros.

Desde el punto de vista psicoterapéutico, es importante trabajar sobre todos estos aspectos, ayudándoles a establecer metas realistas en la vida cotidiana, en las relaciones familiares, laborales y sociales, y con una actitud fundamentalmente integradora de las experiencias del pasado.

Algunos manifiestan su deseo de no acudir al seguimiento postquirúrgico, alegando que es doloroso hacerlo, porque les recuerda una etapa que intentan olvidar. La psicoterapia debe tener en cuenta este aspecto, trabajando con la continuidad de la historia personal y no con la disrupción producida por el acto quirúrgico. Es necesario articular una integración de todos los aspectos de la biografía, estableciendo una continuidad de la vida. La construcción de la personalidad pasa por el género, pero también engloba otros aspectos y abarca la totalidad de la persona (32). Se trabaja la identidad sexual como un proceso de construcción y no como algo dado por la naturaleza (32). Esa experiencia coherente en tiempo y espacio que constituye nuestra identidad se construye y reconstruye constantemente en el curso de nuestras vidas, sobre la base de nuestra interacción con nuestros familiares, amigos, compañeros de trabajo, etc. Los «otros» son co-constructores de nuestra identidad.

En el proceso de reasignación sexual, el cuerpo es modificado por métodos hormonales y/o quirúrgicos con el objeto de transformar el aspecto físico. Esta transformación culmina un proceso que no sólo abarca al cuerpo. También se desarrollan características psíquicas y sociales definidas estereotipadamente como femeninas o masculinas. Se trabajan movimientos, posturas, gestos, poses, actitudes, vestimentas, maquillaje, peinados, modificación voluntaria de la voz. Pero no sólo cambia el cuerpo, sino también el sentido del cuerpo y la interrelación con el medio, que también se modifica. El trabajo y la transformación del cuerpo son evidentes, visibles. Son procesos de autotransformación, caminos sin retorno respecto al género, por lo que se puede decir que el cuerpo no es un mero espejo de la sociedad o la cultura en la que vivimos, sino que sobre todo es un agente (28).

Muchas de las personas transexuales esperan que todo se juegue en el nivel del cuerpo y no de la mente. A veces, aceptan la propuesta de acudir a psicoterapia porque la consideran parte del programa y un paso más para conseguir su obje- 
DEBATES E INFORMES

tivo de que se les restituya su verdadero cuerpo (32). Trabajando con personas transexuales, se escucha con frecuencia la expresión de haber sido objeto de un «error de la naturaleza», error que debe ser corregido.

En la Unidad de Trastornos de identidad de Género, constatamos la enorme diversidad y pluralidad que caracteriza al tema de la identidad. Las identidades son tan plurales y diversas como lo son los estilos de vida que a ellas están asociados. Algunas de estas realidades responden a transformaciones rupturistas que anuncian nuevos tiempos y nuevas realidades muy diversas que no pueden ser entendidas desde el modelo dicotómico que manejamos. Cada vez con mayor frecuencia se observan situaciones y realidades mixtas con mezclas de valores y prácticas definidas socialmente como masculinas y femeninas. Estas realidades se hacen mucho más patentes cuando se produce un cambio geográfico, en procesos de emigración, que permiten tomar conciencia de la contextualidad y relatividad de los conceptos que manejamos como naturales o ahistóricos (28). Se trata de un proceso de cambio, de desidentificación respecto a las formas heredadas, de creación de nuevas imágenes, valores y prácticas institucionales, producen rupturas y configuraciones de nuevas identidades y modelos emergentes de género (33).

Es importante señalar el poder del cuerpo que va mucho más allá del intercambio sexual y «que se constituye en una forma de alegría, de júbilo, de lente de escrutinio y evaluación propias, de conocimiento de uno mismo en su capacidad de sentir, conectar física, psíquica, emocional e intelectualmente con otros seres humanos» (28).

\section{Conclusiones}

1. Las personas transexuales nos hacen pensar acerca de que la identidad es también un proceso subjetivo y depende del punto de vista de quienes la sienten. Sin embargo, sí es posible construir un mínimo común identitario. El acuerdo sobre la identidad surge cuando se construye una subjetividad común. Cuando esto ocurre, se puede hablar de cultura o subcultura (27).

2. Al ser subjetiva, toda identidad es plural. Al ser heterogéneas, sus límites no son nítidos. Vivimos una época en la que coexisten una gran variedad de maneras de experimentar la masculinidad y la feminidad. Sin embargo, las fronteras persisten socialmente y están profundamente interiorizadas. Ser varón o ser mujer siguen siendo identidades demasiado cerradas al cambio (27).

3. Resulta paradójica la posición de las personas transexuales que formulan una doble y contradictoria demanda: por un lado, se sitúan más allá de la biología sosteniendo la primacía del reconocimiento simbólico y por otro, reclaman una modificación de su cuerpo pretendiendo obtener completamente de la biología, 
una prueba de la verdad de su discurso, demostrando la insuficiencia de toda referencia simbólica (13).

4. Las personas transexuales plantean otra importante contradicción: por un lado, de hecho, cuestionan el sistema dicotómico, lo ponen en tela de juicio. Con su existencia, denuncian su insuficiencia para entender una realidad que desborda las coordenadas construidas por nuestra cultura. Anticipan, sugieren otras posibilidades, etc., pero, por otro, anhelan convertirse exactamente en el otro, proporcionando una imagen de sí mismos polarizada. Necesitan ser reconocidos como hombres o como mujeres. El cuerpo y el aspecto externo son la primera tarjeta de presentación y tienen un papel muy importante en la relación con el entorno. La gestión de la apariencia de estas personas tiene estrecha relación con las definiciones sociales de lo que es un hombre o una mujer. Sin embargo, la manera en que cada una de estas personas vivencia esa identidad, no es homogénea, sino compleja, diversa y se resiste a las clasificaciones dicotómicas del tipo masculino/femenino. A veces resulta difícil nombrar, dar un nombre a lo que se ve, y da la sensación de que carecemos de palabras para denominar lo que sucede (28).

5. Trabajar en el tema de la Transexualidad, es trabajar con personas que a veces producen incomodidad y que nos obligan a pensar en una serie de cuestiones que habitualmente no revisamos: el sistema de géneros que hemos construido y que manejamos diariamente, nuestro vocabulario, a ser muy cuidadosos con la asignación sexual de nuestras palabras, a preguntarnos sobre nuestros vínculos con nuestro sexo biológico, en la mención que aparece en nuestra acta de nacimiento o en nuestro documento de identidad, con nuestro sentimiento de pertenencia a uno y otro sexo, con nuestra sexualidad (13).

6. Necesitamos claves nuevas y nuevos modelos de conocimiento, nuevas metáforas, para aproximarnos a tema tan complejo como es el de las relaciones entre género e imagen corporal. Es necesario revisar los modelos dicotómicos que manejamos. Sólo así podemos trabajar en el tema de la transexualidad. De ese modo, podremos identificar las prácticas innovadoras que se están dando de hecho en nuestra sociedad y en nuestra cultura. Se necesitan estudios que incorporen modelos que permitan dar cuenta de la diversidad y el dinamismo de la identidad, de sus rupturas y transgresiones, de la interrelación entre representaciones y prácticas concretas, entre contextos sociopolíticos y vivencias, que tengan en cuenta los macro y micro contextos en los que se mueven las personas transexuales, pero que también tengan en cuenta la acción modificadora de la experiencia de esas personas (28).

7. La tarea más importante del psicólogo y del equipo multidisciplinar que trabaja con personas transexuales es, sin duda, ayudar al paciente a vivir lo más cómodamente posible con un rol y un cuerpo de un determinado sexo, revisando las estrategias habitualmente utilizadas para resolver los problemas, definir y trabajar los conflictos y ayudarle a actuar con el mayor realismo respecto a la actividad 
DEBATES E INFORMES

laboral y a las relaciones sociales (34). Sin duda, el éxito de la tarea recae no sólo en las creencias de la persona que consulta, acerca de en qué consiste ser un hombre o una mujer, sino también en las creencias de los mismos psicólogos que trabajan con ellos, y que por proceder de la misma sociedad y momento histórico, manejan idénticos modelos en relación con la identidad (21).

\section{BIBLIOGRAFÍA}

(1) «Informe preliminar. Problemática de salud ligada a la transexualidad en Andalucía. Posibilidades de abordaje e inclusión entre las prestaciones del sistema sanitario público de Andalucía», Agencia de Evaluación de Tecnologías Sanitarias de Andalucía. Consejería de Salud, Junta de Andalucía, Mayo, 1999.

(2) Das, A.; Wilhelm, M., Tritiya Prakriti: People of the Third Sex: Understanding Homosexuality, Transgender Identity and Intersex Conditions Through Hinduism, Filadelfia, Xlibris Corporation, 2004.

(3) Gyatso, J., «One Plus one Makes Three: Buddhist Gender Conceptions and the Law of the Non-excluded Middle», en History of Religions, 43, University of Chicago Press, 2003.

(4) FernándeZ, J., Cuerpos desobedientes, Buenos Aires, Edhasa, 2004.

(5) BERgero, T.; CANO, G., y otros, «La transexualidad: asistencia multidisciplinar en el sistema público», Revista de la AEN, 2004, 90, pp. 2.828-2.838.

(6) American Psychiatric Association, Diagnostic and Statistical Manual of Mental Disorders, Washington, D. C., 1994 y O. M. S, 4. ${ }^{\text {a }}$ ed.

(7) CIE-10, The ICD-10 Classification of Mental and Behavioural Disorders: Diagnostic criteria for research, OMS, 1992.

(8) The Harry Benjamin International Gender Dysphoria Association (HBIGDA), The Standards of care for Gender Identity Disorders, Minneapolis, HBIGDA, 2001.

(9) The Harry Benjamin International Gender Dysphoria Association (HBIGDA), The Standards of care for Gender Identity Disorders, Minneapolis, HBIGDA, 1998, $5^{\mathrm{a}} \mathrm{ed}$.

(10) Person, E.; Ovesey, L., «The Transexual Sindrome in Males. II Secondary Transsexualism», American Journal of Psychotherapy, 1974, 28.

(11) Stoller, R. J., Sex and Gender, Nueva York, Science House, 1968.

(12) Benjamín, H., The Transsexual Phenomenon, Nueva York, Julian Press, 1966.

(13) Chiland, C., Cambiar de sexo, Madrid, Biblioteca Nueva, 1999.

(14) Сово Bedia, R., Diez palabras clave sobre mujer, Estella, Género/ Amorós/ C. Verbo Divino/, 1995.

(15) Martín Casares, A., Antropología del género. Culturas, mitos y estereotipos sexuales, Madrid, Cátedra, 2006.

(16) MoORe, H., Antropología y feminismo, Madrid, Cátedra, 1999.

(17) GOOD, L.; BYRon, J., Medicina, racionalidad y experiencia. Una perspectiva antropológica, Barcelona, Bellaterra, 2003.

(18) Gómez, E.; Esteva, I. (eds.), Ser transexual. Dirigido al paciente, a su familia, y al entorno sanitario, judicial y social, Barcelona, Glosa, 2006.

(19) SElliPI, T., «¿De qué hablamos cuando hablamos de factores en salud?» en Comelles, J.; Perdiguero, E. (eds.), Medicina y cultura. Estudios sobre Antropología y Medicina, Barcelona, Bellaterra, 2000, pp. 249-275. 
(20) Gooren, L., «El transexualismo, una forma de intersexo», en BECERRA-FERNÁNDEZ, A., Transexualidad. La búsqueda de una identidad, Madrid, Díaz de Santos, 2003.

(21) Garaizábal, C., «Evaluación y consideraciones psicológicas», GómEz, E.; Esteva, I. (eds.), en Ser Transexual, Barcelona, Glosa, 2006.

(22) COHEN, P.; VAN Gooren, S., «Sex Reassignment of Adolescent Transsexuals: A FollowUp Study», J. Am. Acad. Child Adolesc. Psychiatry, 1997, 36 (2), pp. 263-276.

(23) Roscoe, W., «How to Become a Berdache: toward a Unified Analysis of Gender Diversity», en Herdt, G. (ed.), Third Sex, Third Gender, Beyond Sexual Dimorphism in Culture and History, Nueva York, Zone Books, 2006.

(24) NANDA, S., «Hijras: an Alternative Sex and Gender Role in India», en HerdT, G. (ed.), Thrid Sex, Third Gender, Nueva York, Zone Books. 1994.

(25) MatThew, J.; HerTer, D., «Eunucos and Postgender and Transgressive Sexualities», Journal of the Study of the New Testament, 28, 2005.

(26) ButLer, J., Gender Trouble. Feminism and the Subversión of Identity, Nueva York, Routledge, 1990.

(27) Viñuales, O.; Guasch, O., Sobre el futuro de la identidad, Madrid, Reverso, 2000.

(28) Esteban, M. L., Antropología del cuerpo, Barcelona, Bellaterra, 2004.

(29) SLUZKI, C., La red social: frontera de la práctica sistémica, Barcelona, Gedisa, 1996.

(30) Csordas, T. (ed.), Embodiment and Experience. The Existencial Ground of Cultura and Self, Cambridge, Cambridge University Press, 1994.

(31) HARAWAY, D., «Manifiesto para Cyborgs: ciencia, tecnología y feminismo socialista a finales del siglo XX», en Ciencia, cyborgs y mujeres. La reinvención de la naturaleza, Madrid, Cátedra, 1991.

(32) Bergero, M. T.; Cano Oncala, G., «La Asociación Internacional Harry Benjamín de disforia de género, el papel del profesional de salud mental y el test de la vida real», en GómEz GIL, E.; Esteva de Antonio, I. (eds.), Ser Transexual, Barcelona, Glosa, 2006.

(33) Del Valle, T. (coord.) Apaolaza, J. M., y otros, Modelos emergentes en los sistemas y las relaciones de género, Madrid, Narcea, 2002.

(34) CANO, G.; BERgERO, T., «Intervención psicológica en una unidad de trastornos de la identidad de género: un desafío sanitario», en REMOR, E.; ARRANZ, P.; UlLA, S. (eds.), El psicólogo en el ámbito hospitalario, Madrid, Desclèe de Brouwer, 2003.

* Trinidad Bergero Miguel. Psicóloga. Unidad de Trastornos de Identidad de Género. Hospital Carlos Haya. Málaga; Susana Asiain Vierge. Licenciada en Antropología. Enfermera. Hospital de Navarra; Isolde Gorneman Schaffer. Médico. Epidemióloga. Francisco Giraldo Ansio; José Lara Montenegro. Cirujanos Plásticos. Unidad de Trastornos de Identidad de Género. Hospital Carlos Haya. Málaga; Isabel Esteva de Antonio. Endocrinóloga. Unidad de Trastornos de Identidad de Género. Hospital Carlos Haya. Málaga; Marina Gómez Banovio. Psiquiatra. Unidad de Trastornos de Identidad de Género. Hospital Carlos Haya. Málaga.

Artículo elaborado en el marco del Proyecto de Investigación I+D+I del Instituto de la Mujer y llevado a cabo conjuntamente por la Unidad de Trastornos de Identidad de Género del Hospital Carlos Haya de Málaga y la Facultad de Antropología de la Universidad de Granada.

No Expte: 97/06. Ministerio de Trabajo y Asuntos Sociales.

Correspondencia: Trinidad Bergero Miguel. berg7679@hotmail.com. C/ Las Palmeras, 4. 29018 Málaga.

** Fecha de recepción: 2-IX-2007 (aceptado el 28-XI-2007). 